\title{
Treatment of heavy menstrual bleeding associated with uterine leiomyoma with the levonorgestrel-releasing intrauterine system
}

\author{
O. Shawki $\cdot$ Waleed El-Sherbiny $\cdot$ Waleed Saber • \\ Ahmed Askalany • Ashraf El-Daly
}

Received: 30 December 2008 / Accepted: 4 March 2009/Published online: 21 April 2009

(C) Springer-Verlag 2009

\begin{abstract}
Heavy menstrual bleeding is an increasingly common health problem. The levonorgestrel-releasing intrauterine system (LNG-IUS) has been advocated for the treatment of heavy menstrual bleeding as an alternative to surgery. Based on this established indication and theoretical potential of targeted endometrial solution of myoma-related bleeding, we evaluated the efficiency of LNG-IUS in treating selected cases presenting with heavy menstrual bleeding in the presence of uterine leiomyomas. This study included 68 patients with history of heavy menstrual bleeding and ultrasound-proved submucous uterine leiomyoma. Uterine bleeding was done by the semiquantitative pictorial blood loss assessment score. The 5-dimensional EuroQol was chosen as the primary measure of effectiveness on healthrelated quality of life. Patients were re-evaluated at 3, 6, 9, and 12 month. After 12 months of follow-up, a significant reduction of menstrual flow was observed up to amenorrhea (in 11 cases). No significant effect for the LNG-IUS on the volume of fibroids or the uterine volume was observed. A significant improvement of the quality of life (measured by EQ index) was observed. Side effects were reported in ten cases. Eight cases required removal of the IUS for different reasons. So this study shows that in selected cases of uterine leiomyomas, Mirena LNG-IUS is an effective therapy directed towards the local steroid environment of the
\end{abstract}

\footnotetext{
O. Shawki $(\bowtie) \cdot$ W. El-Sherbiny $\cdot$ W. Saber $\cdot$ A. Askalany $\cdot$

A. El-Daly

Department of Obstetrics and Gynecology, Faculty of medicine,

Cairo University,

Giza, Egypt

e-mail: osamashawki@yahoo.com

O. Shawki $\cdot$ W. El-Sherbiny $\cdot$ W. Saber $\cdot$ A. Askalany $\cdot$ A. El-Daly

Al Ebtesama Hopsital,

10 Aboul Magd Amer Street, Heliopolis,

Cairo, Egypt
}

endometrium for control of the bleeding with no valuable effect on the myomas themselves.

Keywords Heavy menstrual bleeding · Uterine leiomyoma . Levonorgestrel-releasing intrauterine system

\section{Introduction}

Heavy menstrual bleeding is an important cause of ill health in women worldwide. About one third of women report heavy menstrual bleeding at some time in their lives [1]. heavy menstrual bleeding is the presenting symptom among the majority of women who undergo hysterectomy [2, 3], and recent data suggest that heavy menstrual bleeding is an increasingly common health problem [4].

Uterine myomas are clinically apparent in $25-50 \%$ of women. They are treated only when they cause symptoms (abnormal uterine bleeding, pelvic pain, and/or pressure). Effective treatment strategies are limited by a narrow understanding of the pathogenesis of this disease. Current strategies for dealing with this problem fall into two broad categories of therapy. The first is surgical therapy, traditionally hysterectomy, myomectomy, or endoscopic procedures aiming for the complete or partial removal of the leiomyomas. The second strategy for the management of leiomyomas is manipulation of the steroid hormone environment. The most widely used agent is gonadotrophin-releasing hormone agonist, but long-term therapy is prohibited because significant hypo-estrogenic effects on bone can lead to osteoporosis [5]. Other therapies include high-dose progestin therapy, danazol and gestrinone, and oral contraceptives to act on the endometrium to produce atrophy [6]. Therapies directed against the endometrium are supported by data which suggest that in some patients the endometrial lining 
overlying submucosal fibroids are abnormal [7]. Several innovative options emerged as surgical alternatives to avoid hysterectomy in the last decade. The levonorgestrel-releasing intrauterine system (LNG-IUS; Mirena) has been extensively studied in the treatment of dysfunctional heavy menstrual bleeding [8].

The levonorgestrel-releasing intrauterine system (Mirena) has been advocated for the treatment of heavy menstrual bleeding as an alternative to surgery. The LNG-IUS is an intrauterine system that releases $20 \mu \mathrm{g}$ of levonorgestrel every $24 \mathrm{~h}$ over 5 years. The LNG-IUS was developed during the 1980s. The estimated number of current LNG-IUS users worldwide is more than 4 million in approximately 100 countries [9].

The LNG-IUS reduces menstrual blood loss (MBL) more than tranexamic acid [10], nonsteroidal anti-inflammatory drugs, danazol, oral progestogens, combined oral contraceptives [11], or long-term norethisterone [12]. No difference in patient satisfaction or health-related quality of life (HRQOL) has been found between the LNG-IUS and endometrial destruction, and both are effective in reducing MBL $[13,14]$. However, data regarding the impact of LNGIUS on myoma-related heavy menstrual bleeding are very scarce and inconsistent at the moment.

Based on the established indication of LNG-IUS in treatment of dysfunctional heavy menstrual bleeding and the theoretical potential of targeted endometrial solution of myoma-related bleeding, we evaluated the efficiency of LNG-IUS in treating selected cases presenting with heavy menstrual bleeding in the presence of uterine leiomyomas.

\section{Material and methods}

The study included 68 patients, aged between 35 and 50 years. The patients were recruited along 2 years. Inclusion criteria included: history of heavy menstrual bleeding over the last 6 months and ultrasound-proved submucous uterine leiomyoma. An informed consent was obtained from all women. The study was designed as an open, therapeutic study with LNG-IUS. Previous attempts at conservative medical treatment were reported in 37 cases. The therapy included sequential progestagen and tranexamic acid tablets for 6 months in most of these patients. Each patient was evaluated on enrolment then after 6 and 12 months following insertion of the LNG-IUS.

Exclusion criteria were breast feeding, current or recent pelvic inflammatory disease, abnormal Papanicolau smear, known endometriosis, breast cancer, a previous history of deep venous thrombosis, thromboembolism or liver disease, and hormone therapy during the last 3 months preceding the study. An endometrial biopsy was performed in all cases.
Initial evaluation included complete medical and obstetrical history including previous contraceptive methods used and complete general and pelvic examination. Laboratory investigations were done to exclude medical diseases and determine the basic level of $\mathrm{Hb}$. Transvaginal ultrasonographic (TVS) examination was done using a General Electric Logiq $500 \mathrm{MD}$ device, with an endovaginal 7.5 $\mathrm{MHz}$ probe. Ultrasound evaluation included assessing the uterus, number and position of leiomyomas, and the status of the ovaries. Volume of leiomyomas was calculated after the "prolate ellipsoid volume": $V=\mathrm{d} 1 \times \mathrm{d} 2 \times \mathrm{d} 3 \times 0.523$, where $\mathrm{d} 1, \mathrm{~d} 2$, and $\mathrm{d} 3$ represent three diameters measured on sagittal and transversal sections of the uterus. Previous therapies for heavy menstrual bleeding, in terms of duration, efficiency, and cause for quitting were recorded.

\section{Quantification of uterine bleeding}

This was done by the semiquantitative pictorial blood loss assessment score (PBAC) [15], completed by all subjects over a screening period of 2 months before enrolment. The score is recorded on a chart on which the patient registers the number of sanitary pads or tampons used each day, the degree of soiling on each pad or tampon, the number and size of clots passed, and episodes of flooding. A monthly score of $>75$ on this chart, corresponding to a blood loss of $>60 \mathrm{~mL}$, was regarded as excessive bleeding.

\section{Health-related quality of life}

The 5-dimensional EuroQol (EQ-5D) $[16,17]$ was chosen as the primary measure of effectiveness because it provides a single numeric score for HRQOL and is universally used. The EQ-5D assesses five dimensions of HRQOL: mobility, self-care, usual activities, pain or discomfort, and anxiety or depression. Each dimension is measured on a three-point ordinal scale where a higher score corresponds to a worse health state (no limitation, some limitation, and greatest limitation in HRQOL). A strength of the EQ-5D is that through their response, patients can effectively locate themselves into one of 245 possible health states for which a global value (i.e., a "utility score") is assigned. A utility score of 1 corresponds to the highest degree of HRQOL, and score of 0 is compatible with a level of HRQOL equivalent to death. A negative score indicates a state of HRQOL that is worse than death.

At 6 and 12 months of follow-up, we studied:

1 Changes in the bleeding pattern, assessed with the PBAC

2 Changes in the volume of uterus and fibroids, assessed by ultrasound

3 HRQOL score 
4 Side effects, the positive effects, and method acceptability

5 The method acceptability, assessed by percentage of patients asking for LNG-IUS removal in the first year

\section{Statistical analysis}

Data were analyzed using SPSSwin statistical package version 12. Numerical data were expressed as mean \pm SD or median and range. Analysis of variance with repeated measures was used to compare the condition of the studied group after 6 and 12 months with their baseline criteria. Probability ( $p$ value) less than 0.05 was considered significant.

\section{Results}

Demographic and baseline clinical data of the studied group are shown in Table 1. No complications occurred during the insertions of the IUSs. Insertion of the intrauterine system could not be achieved in a woman having low-lying submucosal fibroid identified on ultarasonography. Dilatation of the cervix was needed in two cases. Six patients reported postinsertion spotting which was self-limited within 2 days. Ten patients reported generalized symptoms, including abdominal pain $(n=4)$, breast tenderness $(n=3)$, headache $(n=2)$, acne $(n=2)$, and mood changes $(n=1)$. Six patients were lost to follow-up after 6 months, and another three were lost at 12 months. In addition, eight cases requested IUS removal within the study period. The reason and timing for discontinuation of treatment is presented in Table 2. Accordingly, we commented on 57 cases at 6 months and 50 at 12 months.

Changes of the amount of blood loss and bleeding pattern are shown in Tables 3 and 4. A significant drop of the amount of menstrual bleeding was observed after 6 and 12 months using the PBAC. Duration of menstruation had also decreased significantly during the two follow-up visits.

On the other hand, there was no significant effect for the LNG-IUS on the volume of fibroids or the uterine volume in general as measured by TVS. This was the case during the two follow-up visits at 6 and 12 months (Table 5).

A significant improvement of the quality of life was observed after 6 and 12 months. This is demonstrated in Fig. 1. The utility score (EQ index) increased from $0.56 \pm$ 0.12 at baseline to $0.78 \pm 0.13$ at 6 months and $0.79 \pm 0.08$ at 12 months.

\section{Discussion}

Three scores are used frequently in the literature to assess the efficiency of a treatment of heavy menstrual bleeding: the total menstrual fluid loss, the menstrual blood loss, and pictorial blood loss assessment chart-also called Higham chart. We used the last one, which, although subjective, is the most widely used in the literature studies that analyzed the LNG-IUS efficiency for treating heavy menstrual bleeding, therefore allowing us to compare our results with those from other authors. Initial studies demonstrated a sensitivity and specificity of $>80 \%$ for a PBAC score of $>100$ [5]. On the other hand, Reid et al. [18] have reported that there is no correlation between the observed PBAC and actual measured blood loss.

With improved technology in sanitary protection, there will be a change in the reliability of this score over time, though it is suggested that such a scoring system is an unreliable tool that adds little to the subjective complaint. The advantage of PBAC is that it does provide semiobjective comparative information in the absence of a more valid and reliable test.

We reported a reduction of PBAC during the follow-up visit at 12 months of $88.3 \%$. Eleven women $(16.2 \%)$ were amenorrheic at 12 months. Other authors reported figures between $69 \%$ and $90 \%$ reduction of menstrual flow measured by PBAC [19-25]. Similar results were reported by Hickey and Farquhar [26], where in women with ovulatory dysfunctional uterine bleeding, Mirena system has been shown to produce an $86 \%$ reduction in objectively measured menstrual blood loss at 3 months and a $97 \%$ reduction at 12 months, with amenorrhea in $50 \%$ of women at 12 months.
Table 1 Demographic and baseline clinical characteristics of the studied group

\begin{tabular}{ll}
\hline Characteristic & Value \\
\hline Age (mean \pm SD) & $43 \pm 3.6$ \\
Initial PBAC score [median (range)] & $325(110-650)$ \\
Initial uterine volume (cmc) (mean \pm SD) & $123 \pm 24.1$ \\
Number of fibroids per case [median (range)] & $1(1-7)$ \\
Total initial volume of fibroids per case [median (range)] & $12.4(1.5-220.6)$ \\
Births number [median (range)] & $2(0-4)$ \\
Abortion number [median (range)] & $2(0-10)$ \\
\hline
\end{tabular}


Table 2 Reasons for discontinuation of use of levonorgestrel intrauterine system and histological diagnosis at hysterectomy

${ }^{a}$ The patient refused surgery

The oligomenorrhea or amenorrhea in LNG-IUS users is hypothesized to be mediated by suppression of endometrial proliferation [27] as well as endometrial vascular changes that include thickening of the arterial walls, suppression of spiral arterioles, and capillary thrombosis [28].

Several Doppler flow studies evaluated the hemodynamic changes in LNG-IUS users. Pakarinen et al. [29], who examined the impedance to uterine blood flow before and after the insertion of LNG-IUS in ten women of fertile age, did not find any change in the uterine pulsatility index (PI). Jarvela et al. [30] demonstrated an increase in the main uterine artery PI in the mid-luteal phase but not on the first day of menstruation and that the extent of increase in the PI correlated with serum levonorgestrel concentration. Zalel et al. [31] found that Doppler flow did not reveal any significant change in the uterine artery, whereas there was a marked reduction in the subendometrial blood flow in the LNG-IUS users. This observation was reinforced by the significant reduction in endometrial thickness in these patients.

According to these studies, the main effect of the LNG-IUS is due to its local progestational effect (i.e., no change in uterine blood flow along with marked reduction in spiral artery blood flow and endometrial width). These hemodynamic changes correlate well with the morphological changes in the endometrial spiral arterioles and capillaries [32]. These authors have demonstrated a remarkable transformation of the endometrium, mediated by the local levonorgestrel delivery and manifested by extensively decidualized and atrophic morphology, significant reduction in steroid receptor content, resulting in the alteration of many progesteroneregulated locally acting mediators [32].

Table 3 The changes in pictorial blood loss assessment chart score during follow-up visits

\begin{tabular}{lrrrr}
\hline Time & Median & Minimum & Maximum & $p$ value \\
\hline Baseline $(n=67)$ & 325 & 110 & 650 & \\
6 months $(n=57)$ & 54 & 0 & 90 & $<0.01$ \\
12 months $(n=50)$ & 38 & 0 & 80 & $<0.01$ \\
\hline
\end{tabular}

They have also stated that this phenomenon is independent of ovarian function and circulating sex steroid concentrations. In addition it should be acknowledged that levonorgestrel has androgenic and anti-estrogenic activities on the endometrium that result mainly from downregulation of estrogenic receptors [32]. French et al. [33] and Zalel et al. [31] stated that the amenorrhea in LNG-IUS users is benign, end-organ suppression of bleeding, and associated with normal estrogen levels

We notice that in all the cited studies, authors described an important decrease of blood loss at 12 months follow-up. Also, the initial score was similar in patients with bleeding induced by dysfunctional uterine bleeding or with fibroids (as detailed in the studies of Grigorieva [21] and of Mercorio [22]), and the decreasing effect was significant in all cases. This proves that LNG-IUS reduces bleeding originating mainly from the endometrium and not by stopping fibroid proliferation.

In a prospective controlled trial, Soysal and Soysal [8] evaluated the efficacy of LNG-IUS insertion in menorrhagic patients who have a myomatous uterus $<380 \mathrm{ml}$ at ultrasonography. They reported statistically significant decreases in menstrual blood flow and increases in hemoglobin values. They concluded that, in selected cases of myoma-induced heavy menstrual bleeding, LNG-IUS was very efficient in reducing menstrual blood loss and increasing hemoglobin values. They demonstrated that the effectiveness of LNG-IUS in this setting is equivalent to results obtained as compared to thermal balloon ablation.

In our study, initial uterine volumes were within $300 \mathrm{ml}$. Both uterine and the leiomyoma volume evolution were relatively stable after 12 months follow-up. Considering the

Table 4 The evolution regarding menstrual duration in days during follow-up

\begin{tabular}{lcc}
\hline Time & Mean \pm SD & $p$ value \\
\hline Baseline $(n=67)$ & $6.7 \pm 3.1$ & \\
6 months $(n=57)$ & $4.4 \pm 1.2$ & $<0.01$ \\
12 months $(n=50)$ & $3.2 \pm 0.9$ & $<0.01$ \\
\hline
\end{tabular}


Table 5 The declared side effects at follow-up

\begin{tabular}{lcc}
\hline Side effects & Number & \% from total \\
\hline Weight gain & 6 & 8.9 \\
Insomnia & 2 & 2.9 \\
Headache & 3 & 4.5 \\
Pelvic pain & 4 & 5.9 \\
Breast tenderness & 2 & 2.9 \\
Bloating & 3 & 4.5 \\
Ovarian cysts appearing during follow-up & 11 & 16.4 \\
\hline
\end{tabular}

20-30\% natural increase rate per year for fibroids - as shown by prospective studies [34], we can estimate that LNG-IUS reduces their size or at least inhibits their growth. The variation was not statistically significant ( $p$ value of 0.46 for uterine volume and 0.37 for total volume of fibroids). Several studies support this hypothesis [21, 22, 34]. In all studies, the uterine and fibroid volumes did not increase under IUS-LNG.

Striking reduction in heavy menstrual bleeding in women with uterine myomas after the use of LNG-IUS prompted Maruo et al. [35] to characterize the effects of progesterone on the growth and apoptosis of uterine leiomyoma cells. Their results suggested that progesterone has dual actions on uterine leiomyoma growth: one is to stimulate leiomyoma cell growth and survival through upregulating EGF and $\mathrm{Bcl}-2$ protein expression as well as down-regulating TNF-alpha expression in those cells, and the other is to inhibit leiomyoma cell growth through downregulating IGF-I expression in those cells. This may explain why the size of uterine myomas during use of LNG-IUS increases in some but decreases in other instances. This may also explain why the size of uterine myomas during pregnancy does not increase despite the overwhelming increase in circulating concentrations of sex steroid hormones.

In our opinion, the strong endometrial suppression is the principal mechanism explaining the effect on menstrual blood loss of the IUS in cases of leiomyoma. This was the same suggestion of Wildemeersch and Schacht [36] in their

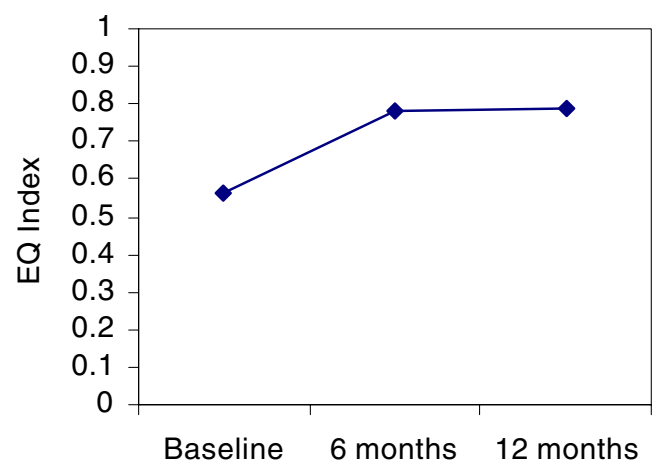

Fig. 1 Baseline and follow-up for patients with heavy menstrual bleeding using LNG-IUS study of premenopausal cases with fibroids. In their series, two failures were observed: one with large endometrial polyp and the other with submucous myoma. This failure points to the fact that it is unlikely to treat local lesions with the IUS.

In a study of 12 women with uterine fibroids and severe menstrual bleedings, post treatment ultrasonography revealed endometrium thickness of no more than $4 \mathrm{~mm}$ and no change in the fibroids volume [37].

Mercorio et al. [38] reported different results. They evaluated the effectiveness of LNG-IUS in the treatment of myoma-related heavy menstrual bleeding in 19 patients. They reported statistically significant reduction of PBAC score, but persistent heavy menstrual bleeding, defined as a monthly PBAC score of 100 or higher, was observed at 12 months in 14 patients, whereas only one woman was amenorrheic, and four were hypomenorrheic.

During the past two decades, ideas of health have transformed from ones that focus on illness to ones that consider patient well-being and quality of life (QOL). Currently, QOL instruments that integrate clinical and economic indices serve as a key to understanding treatment outcomes. We used the EQ-5D as a measure of quality of life in this study. Initially, patients show a low EQ index of $0.56 \pm 0.12$ during the evaluation visit. A similar figure $(0.55)$ was reported for cases of heavy menstrual bleeding in another series

The strong effect of LNG-IUS on menstrual blood loss offers an important health benefit and improvement in quality of life, particularly in women with heavy bleeding and anemia, as other treatment modalities are less effective, more costly, more invasive, or not readily available.

We can conclude that, in cases of uterine leiomyomas, the use of LNG-IUS is a type of selective therapy directed towards the local steroid environment of the endometrium for control of the bleeding problem confronted in these cases. No valuable effect was observed on the myomas themselves, and this must not be the target of this therapeutic modality. Control of heavy menstrual bleeding is by itself a crucial target for any type of therapy of leiomyomas. It will be very beneficial for premenopausal women to get rid of heavy menstrual bleeding for 5 years 
after a single session for insertion of a LNG-IUS and thus avoid a major surgical intervention, myomectomy, or hysterectomy.

\section{Conclusion}

In selected cases of uterine leiomyomas, LNG-IUS is an effective therapy directed towards the local steroid environment of the endometrium for control of the bleeding with no valuable effect on the myomas themselves.

\section{References}

1. Market Opinion and Research International (MORI) (1990) MORI Women's Health in 1990 [research study conducted on behalf of Parke-Davis Research Laboratories]. MORI, London, England

2. Vuorma S, Teperi J, Hurskainen R, Keskimäki I, Kujansuu E (1998) Hysterectomy trends in Finland in 1987-1995 — a register based analysis. Acta Obstet Gynecol Scand 77:770-776

3. Stirrat GM (1999) Choice of treatment for menorrhagia. Lancet $353: 2175-2176$

4. Royal College of Obstetricians and Gynaecologists (RCOG). The initial management of menorrhagia. London, England: RCOG; 1998. RCOG Evidence-Based Guidelines No. 1

5. Stewart EA, Friedman AJ (1992) Steroidal treatment of myomas: preoperative and longterm medical therapy. Semin Reprod Endocrinol 10:344-357

6. Rein MS, Friedman AJ (1988) Medical therapy for uterine leiomyomata. In: Barbieri RL, Schiff I (eds) Reproductive therapeutics. Alan R.Liss Inc., New York, NY, USA, pp 199-222

7. Stewart EA, Nowak RA (1996) Leiomyoma-related bleeding: a classic hypothesis updated for the molecular era. Hum Reprod Update 2(4):295-306

8. Soysal S, Soysal ME (2005) The efficacy of levonorgestrelreleasing intrauterine device in selected cases of myoma-related menorrhagia: a prospective controlled trial. Gynecol Obstet Invest 59(1):29-35

9. Hurskainen R, Teperi J, Rissanen P et al (2004) Clinical outcomes and costs with the levonorgestrel-releasing intrauterine system or hysterectomy for treatment of menorrhagia: randomized trial 5-year follow-up. JAMA 291:1456-1463

10. Milsom I, Andersson K, Andersch B, Rybo G (1991) A comparison of flurbiprofen, tranexamic acid, and a levonorgestrel-releasing intrauterine contraceptive device in the treatment of idiopathic menorrhagia. Am J Obstet Gynecol 164:879-883

11. Farquhar CM (1992) Management of dysfunctional uterine bleeding. Drugs 44:578-584

12. Irvine GA, Campbell-Brown MB, Lumsden MA, Heikkilä A, Walker J, Cameron IT (1998) Randomised comparative trial of the levonorgestrel intrauterine system and norethisterone for treatment of idiopathic menorrhagia. BJOG 105:592-598

13. Crosignani PG, Vercellini P, Mosconi P, Oldani S, Cortesi I, De Giorgi O (1997) Levonorgestrel-releasing intrauterine device versus hysteroscopic endometrial resection in the treatment of dysfunctional uterine bleeding. Obstet Gynecol 90:257-263

14. Kittelsen N, Istre O (1998) A randomized study comparing levonorgestrel intrauterine system (LNG-IUS) and transcervical resection of the endometrium (TCRE) in the treatment of menorrhagia: preliminary results. Gynaecol Endosc 7:61-65
15. Higham JM, O'Brien PMS, Shaw RW (1990) Assessment of menstrual blood loss using a pictorial chart. Br J Obstet Gynaecol 97:734-739

16. EuroQol Group (1990) EuroQol - a new facility for the measurement of health-related quality of life. Health Policy 16:199-208

17. Ohinmaa S, Sintonen H (1998) Inconsistencies and modeling of the Finnish EuroQol (EQ-5D) preference values. In: Gainer W, Schulenburg G, Piercy J (eds) Discussion Papers of the 15th Annual EuroQol Plenary Meeting; October 1-2, 1998; Hanover, Germany. Hannover: Center of Health Economics and Health System Research, University of Hannover; 57-74

18. Reid PC, Coker A, Coltart R (2000) Assessment of menstrual blood loss using a pictorial chart: a validation study. BJOG 107(3):320-322

19. Hurskainen R, Teperi J, Rissanen P et al (2001) Quality of life and cost-effectiveness of levonorgestrelreleasing intrauterine system versus hysterectomy for treatment of menorrhagia. Lancet 357:273-277

20. Fedele L, Bianchi S, Rafaelli R et al (1997) Treatment of adenomyosis-associated menorrhagia with a levonorgestrel releasing intrauterine device. Fertil Steril 68:426-429

21. Grigorieva V, Chen Mok M, Tarasova M, Mikhailov A (2003) Use of a levonorgestrel_releasing intrauterine system to treat bleeding related to uterine leiomyomas. Fertil Steril 79:1194-1198

22. Mercorio F, De Simone R, Di Spezio Sardo A et al (2003) The effect of a levonorgestrel-releasing intrauterine device in the treatment of myoma-related menorrhagia. Contraception 67:277-280

23. Crossignani PG, Vercellini P, Mosconi P et al (1997) Levonorgestrel-releasing intrauterine device versus hysteroscopic endometrial resection in the treatment of dysfunctional uterine bleeding. Obstet Gynecol 90:257-263

24. Istre P, Trolle B (2001) Treatment of menorrhagia with the levonorgestrel intrauterine system versus endometrial resection. Fertil Steril 76:304-309

25. Kittelsen N, Istre O (1998) A randomised study comparing levonorgestrel-releasing intrauterine system (LNG-IUS) and transcervical resection of the endometrium in the treatment of menorrhagia: preliminary results. Gynaecological Endoscopy 7:61-65

26. Hickey M, Farquhar CM (2003) Update on treatment of menstrual disorders. Med J Aust 178(12):625-629

27. Barbosa I, Olsson SE, Odlind V, Goncalves T, Coutinho E (1995) Ovarian function after seven years' use of a levonorgestrel IUD. Adv Contracept 11:85-95

28. Zhu PD, Luo HZ, Cheng J, Xu RH, Liu YF, Zheng WM, Song GY, Chen JH, Zhou ZH, Wu YM et al (1989) The effect of intrauterine devices (stainless steel ring, copper-T220 and levonorgestrel) on the DNA content in isolated human endometrial cells. Contraception 40:591-604

29. Pakarinen P, Luukkainen T, Laine H, Lahteenmaki P (1995) The effect of local intrauterine levonorgestrel administration on endometrial thickness and uterine blood circulation. Hum Reprod 10:2390-2394

30. Jarvela I, Tekay A, Jouppila P (1998) The effect of a levonorgestrelreleasing intrauterine system on uterine artery blood flow, hormone concentrations and ovarian cyst formation in fertile women. Hum Reprod 13:3379-3383

31. Zalel Y, Shulman A, Lidor A, Achiron R, Mashiach S, Gamzu R (2002) The local progestational effect of the levonorgestrelreleasing intrauterine system: a sonographic and Doppler flow study. Human Reprod 17(11):2878-2880

32. Jones RL, Critchley HO (2000) Morphological and functional changes in human endometrium following intrauterine levonorgestrel delivery. Hum Reprod 15(Suppl. 3):162-172

33. French RS, Cowan FM, Mansour D, Higgins JP, Robinson A, Procter T, Morris S, Guillebaud J (2000) Levonorgestrel-releasing (20 microgram/day) intrauterine systems (Mirena) compared with other methods of reversible contraceptives. Br J Obstet Gynaecol 107:1218-1225 
34. Zsigmond I, Stamatian F, Bondor C (2005) [Levonorgestrel intrauterine device in leiomyoma associated bleeding] (Romanian). Obstetrica Ginecologia 53:145-149

35. Maruo T, Matsuo H, Shimomura Y, Kurachi O, Gao Z, Nakago S, Yamada T, Chen W, Wang J (2003) Effects of progesterone on growth factor expression in human uterine leiomyoma. Steroids 68(10-13):817-824

36. Wildemeersch D, Schacht E (2002) The effect on menstrual blood loss in women with uterine fibroids of a novel "frameless" intrauterine levonorgestrel-releasing drug delivery system: a pilot study. Eur J Obstet Gynecol Reprod Biol 102(1):74-79

37. Starczewski A, Iwanicki M (2000) Intrauterine therapy with levonorgestrel releasing IUD of women with hypermenorrhea secondary to uterine fibroids. Ginekol Pol 71(9):1221-1225

38. Mercorio F, De Simone R, Di Spiezio Sardo A, Cerrota G, Bifulco G, Vanacore F, Nappi C (2003) The effect of a levonorgestrelreleasing intrauterine device in the treatment of myoma-related menorrhagia. Contraception 67(4):277-280 